\title{
NUCLEI: A New Unmanned Aerial Vehicle Early Warning Method for the No-fly Zone
}

\author{
Jiaming Wang, Bailong Yu*, Zhifang Ma, Shenglan Yang, Li Lu and Wei Yang \\ State Grid Sichuan Electric Power Company, Chengdu, China \\ ${ }^{*}$ Corresponding author
}

\begin{abstract}
In order to monitor the unmanned aerial vehicle(UAV) around the no-fly zone, we proposed a new unmanned aerial vehicle Early warning method for the no-fly zone (NUCLEI) which gives an alarm to the UAV around the nofly zone in real time. We utilize the least square method to match with the flight trajectory of the UAV in the current period, and then we use the minimum external rectangle (MERT) method to obtain the MERT of the electronic fence. Finally, we calculate whether the tangent line at the current point of the flight path fits curve will intersect with the electronic fence to make the prediction for if the UAV will enter the no-fly zone.
\end{abstract}

Keywords-UAV; no-flyzone; trajectory prediction; curve fitting

\section{INTRODUCTION}

UAV mainly used for reconnaissance, and surveillance plays a very important role in modern warfare ${ }^{[1-2]}$. In order to ensure the traffic safety of the airspace and prevent the UAVs around the no-fly zone entering into the area, we proposed NUCLEI, which can report an emergency to all the UAV's around no-fly zone timely and ensure flight safety.

\section{RELATED WORK}

In recent years, the anti-collision technology of UA V has been studied in depth. With collision region calculated by collision region using a certain threshold value of distance or time, an UAV dynamic collision regional model based on maneuver information of both UA V and the aerial intruder was presented ${ }^{[3]}$. A modified differential evolution algorithm was proposed to realize formation reconfiguration without collision [4]. SIR particle filter was used to estimate the state of the intruder ${ }^{[5]}$. The paper ${ }^{[6]}$ proposed a collision avoidance algorithm for UA Vs based on the Lyapunov method. The keys to the UAV's early warning for the no-fly zone are the prediction of UAV flight trajectory ${ }^{[7-8]}$ and the difficulty of how to determine whether the UAV will enter the no-fly zone. Trajectory prediction was presented for the movement track of moving target by the linear neural network ${ }^{[9-10]}$. The paper compared the algorithm based on $\alpha / \beta / \gamma$ filtering, Kalman filtering(KF), and interacting multiple ${ }^{[11]}$.An improved KF was proposed to estimate the $4 \mathrm{D}$ trajectory ${ }^{[12]}$, which increased the accuracy of trajectory prediction through real-time estimation of system noise, but large number of training samples, and a slow learning speed existed. The trajectory prediction based on data mining was studied to calculate out the possible movement trajectory by predicting the dynamic behavior of moving objects ${ }^{[13-15]}$. Paper ${ }^{[16]}$ reviewed existing trajectory data mining techniques that required an enormous amount of computing and storage space, which cannot be applied at one time.

Through the further study and research, we proposed a method called the NUCLEI to make a warning for all the UAVs around the no-fly zone from anotherperspective.

\section{THE OVERALL DESIGN SCHEME}

The NUCLEI mainly solves two problems: the prediction for the UAV's flight trajectory and the solution of whether the UA V will enter the no-fly zone. Aiming at the problem, three innovations are proposed: (1) Give up the end coordinate point and add the new coordinate point; (2) Take full advantage of the tangent line of the UAV current movement point to make a prediction; (3) adopt MERT to determine whether the UAV will enter the no-fly zone.

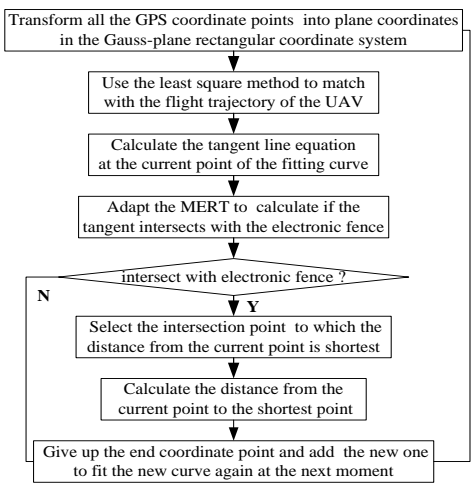

FIGURE I. THE DIAGRAMMATIC SKETCH OF THE NUCLEI 
The flow diagram of the method (MERT) is shown:

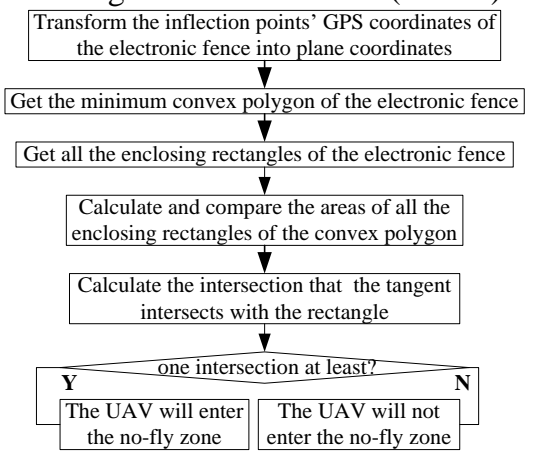

FIGURE II. THE FLOW DIAGRAM OF THE MERT

\section{Flight TRAJECTORY PREDICTION}

We collect some discrete coordinates include the current movement point of the UA V flight, and employ the appropriate curve fitting method ${ }^{[17]}$ to match with the flight trajectory of UA V during this period. At the next moment, we give up the end coordinate point and add the new coordinate to fit the new flight curve, in order to predict the UAV flight trajectory continually. Then by calculating whether the tangent curve at the current point will intersect with the electronic fence (if it is, calculate the current distance of the current coordinate point to the electronic fence), it is determined whether the UAV will enter the no-fly zone.

\section{A. The Curve Fitting of UAV Flight Path}

Due to the unknown of UAV's motion, it is unreliable and unreasonable to predict the flight path of the UAV just depending on the fitting curve at one time, so we must make a forecast for the movement trajectory at each sampling time:

1) transform discrete latitude and longitude coordinates currently into the rectangular plane coordinates in the Gauss plane rectangular coordinate system and store them;

2) use the least square method of curve fitting to fit the flight path of the UAV into a curve to describe its flight path;

3) Give up the end coordinate point and add the new coordinate point to fit the new curve of flight trajectory;

4) Repeat the above steps .

It is feasible to use some discrete coordinate points of the UA V flight in the current period of time to execute on curve fitting by the least squares method ${ }^{[18-20]}$. The error between the fitting curve and the actual data could be very small in order to achieve the better prediction effect of UAV's flight trajectory.

It is found that taking advantage of the five polynomial function to execute on fitting curve have relatively good results after a large nu mber of MATLAB simulation experiments [21-22]. So in this paper, the five polynomial function is used to fit the curve of UAV flight trajectory in the period., and in consideration of the computation complexity and the real-time, we have finally selected 10 discrete data points after a lot of simulation experiments, it means $n$ is equal to 10 . The steps of using the least square method to predict the flight trajectory of $\mathrm{UAV}$ is shown as follows:

1) The first step of the least square method is to take the partial of $E^{\wedge} 2$ with respect to $a j(j=0,1, \ldots, m)$ and can get (1) $(\mathrm{m}=5)$ equations:

$$
\left\{\begin{array}{c}
-2 \sum_{i=1}^{n}\left[y_{i}-\left(a_{0}+a_{1} x_{i}+a_{2} x_{i}^{2}+\ldots+a_{m} x_{i}^{m}\right)\right]=0 \\
-2 \sum_{i=1}^{n}\left[y_{i}-\left(a_{0}+a_{1} x_{i}+a_{2} x_{i}^{2}+\ldots+a_{m} x_{i}^{m}\right)\right] x_{i}=0 \\
\ldots \\
-2 \sum_{i=1}^{n}\left[y_{i}-\left(a_{0}+a_{1} x_{i}+a_{2} x_{i}^{2}+\ldots+a_{m} x_{i}^{m}\right)\right] x_{i}^{m}=0
\end{array}\right.
$$

There will be 6 equations, a0, a1,.., a5 are 6 unknown variables, so the set of equations are solvable.

2) The second step is to deal with them for the normal equations of $\mathrm{a} 0, \mathrm{a} 1, \ldots, \mathrm{a} 5$. The final set of equations is as follows.

$$
\left\{\begin{array}{c}
a_{0} n+a_{1} \sum_{i=1}^{n} x_{i}+a_{2} \sum_{i=1}^{n} x_{i}^{2}+\ldots+a_{m} \sum_{i=i}^{n} x_{i}^{m}=\sum_{i=1}^{n} y_{i} \\
a_{0} \sum_{i=1}^{n} x_{i}+a_{1} \sum_{i=1}^{n} x_{i}^{2}+a_{2} \sum_{i=1}^{n} x_{i}^{3}+\ldots+a_{m} \sum_{i=1}^{n} x_{i}^{m+1}=\sum_{i=1}^{n} x_{i} y_{i} \\
a_{0} \sum_{i=1}^{n} x_{i}^{m}+a_{1} \sum_{i=1}^{n} x_{i}^{m+1}+a_{2} \sum_{i=1}^{n} x_{i}^{m+2}+\ldots+a_{m} \sum_{i=1}^{n} x_{i}^{m}=\sum_{i=1}^{n} x_{i}^{m} y_{i}
\end{array}\right.
$$

3) The third step is to solve the multi-head linear equations and get the polynomial coefficients(a0, a 1,.., a5), then we can get the polynomial function of curve fitting. The Gauss elimination method is used to solve the multiple equations. Finally, the fitting curve which can fit the flight path of the aircraft will be obtained.

\section{B. Prediction of Based on Current Point Tangent Line}

The next challenge after fitting out the flight path is how to determine whether the UAV will enter the no-fly zone, i.e. whether there is an intersection between the electronic fence and the fitting curve of the flight trajectory of the UAV. The general idea will exist two problems: (1)the curve fitted at the current time may change at the next moment, so it is obviously unreasonable to determine whether the UAV will enter the nofly zone only according to the situation that the fitting curve intersects with the electronic fence at the current time directly. (2)Considering the varied shape of the no-fly zone, and the require ment of real-time, the computational complexity of the entire algorithm cannot be too great, which makes the whole prediction is not so easy.

A new idea based on the tangent line of the current point of the UAV flight trajectory was adopted to predict whether UA V will fly into the no-fly zone. In this article from the other point of view, by calculating the tangent line equation of the curve's current point, it can be commendably stated if the UAV has a tendency to fly into the no-fly zone at the current juncture.

According to the trajectory fitting curve and the current coordinate point $\mathrm{G}$ of the $\mathrm{UAV}$, calculate the slope $\mathrm{K}$ of the 
tangent equation of current point, then plug the coordinates of the current point $G(x 0, y 0)$ into $y=k^{*} x+b$, we can calculate the tangent equation of the current point. Let $\mathrm{G}(\mathrm{x} 0, \mathrm{y} 0)$ is the current point of the UAV flight trajectory curve $y=f(x)$, if the derivative of fitting curve in point $G$ exists, the tangent equation of the point $\mathrm{G}$ is:

$$
\mathrm{y}-y_{0}=f^{\prime}\left(x_{0}\right)\left(x-x_{0}\right)
$$

According to the definition of the tangent line, if the curve's tangent line at the point $G$ parallel to the $Y$ axis (that is, the derivative does not exist), the tangent line equation of point $\mathrm{G}$ is: $x=x_{0}$ (4),By the formula (3) and (4) we can get the tangent equation of the current point of the UAV movement trajectory. Calculating whether the tangent intersects with the electronic fence can predict if the UAV will enter the no-fly zone. The following Fig. III shows that the tangent line at the current point is obtained by fitting the curve.

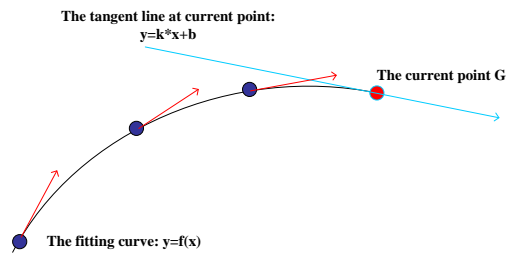

FIGURE III. THE T ANGENT LINE AT THE CURRENT POINT

\section{THE PREDICTION OF THE UAV ENTERING THE NO-FLY ZONE}

In this paper, we put forward MERT to determine whether a straight line intersects with irregular graphics: (1) Transform the latitude and longitude coordinates of all the inflection points of the electronic fence into plane coordinates. (2) Calculate the MERT that contains the electronic fence. (3) Predict if the UAV will enter the no-fly zone by calculating directly whether the tangent line of the UA V flight trajectory at the current point intersects with the MERT of the electronic fence.

\section{A. Obtain the MERT of the Electronic Fence}

Taking into account the problems of redundancy and security, we can find the MERT of the electronic fence to take the place of it, by judging directly if the tangent line of the current point intersects with the MERT to predict whether the UAV will enter the no-fly zone.

We can find the convex hull of the electronic fence to get all the enclosing rectangles, and then compare the area of them ${ }^{[23]}$ to get the MERT of the electronic fence. The specific steps are as follows:

1) Get the minimum convex polygon of the electronic fence We find the minimum convex polygon of the electronic fence by finding the convex hull of the inflection point set of the electronic fence ${ }^{[24]}$.
For three arbitrary points on the plane $\mathrm{p} 1(\mathrm{x} 1, \mathrm{y} 1), \mathrm{p} 2(\mathrm{x} 2, \mathrm{y} 2)$, $\mathrm{p} 3(\mathrm{x} 3, \mathrm{y} 3)$, by calculating the size of the $\mathrm{D}$, it is determined that the loop turns left or right. The size of $\mathrm{D}$ will be gotten by the formula (10):

$$
\mathrm{D}=\left(x_{3}-x_{2}\right) *\left(y_{2}-y_{1}\right)-\left(x_{2}-x_{1}\right) *\left(y_{3}-y_{2}\right)
$$

If $\mathrm{D}<0$, the slope of straight line $\mathrm{p} 1 \mathrm{p} 2$ is less than the slope of straight line $\mathrm{p} 2 \mathrm{p} 3$, so turning left is the right direction; if $\mathrm{D}>0$, the slope of straight line $\mathrm{p} 1 \mathrm{p} 2$ is greater than the slope of straight line p2 p3, so turning right is the right direction; if $\mathrm{D}=0$, it means that they are equivalent, the three points are in one straight line. It is shown as follows:

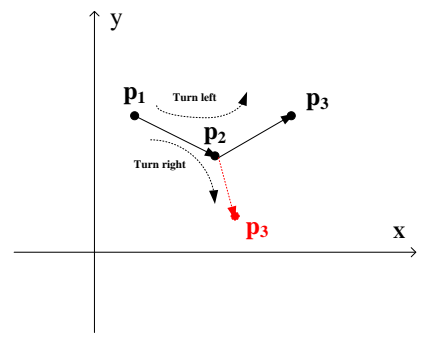

FIGURE IV. THREE ARBITRARY POINTSCONSIST (P1,P2,P3) OF THE LOOP TURNS LEFT OR RIGHT

\section{2) Get all the enclosing rectangles of the electronic fence}

a) Select one side of the convex polygon as the starting side, get the linear equation of the side according to the two vertexes of the edge, and the straight line is denoted by L1, the slope is denoted by $\mathrm{k} 1$;

$b$ ) Find out the point to which the distance from the straight line L1 is farthest from all the vertexes of the convex polygon, which is denoted as px. And find out the linear equation, its slope is also $\mathrm{k} 1$ and it go through the point $\mathrm{px}$, and the straight line is denoted by L2;

c) Note the left endpoint of the selected side as e, and find out the linear equation, its slope is $-1 / \mathrm{k} 1$ and it go through the point e, note the straight line as L3. According to the formula of the distance from a point to a line:

$$
\mathrm{d}=\frac{\left|A x_{0}+B y_{0}+C\right|}{A^{2}+B^{2}}
$$

find out the inflection point of the electronic fence which is on the left of the straight line L3, and to which the distance from the straight line L3 is farthest, it is denoted by py. And then find out the inflection point which is on the right of the straight line L3, and to which the distance from the straight line L3 is farthest, it is denoted by pz. Get the linear equation, its slope is $-1 / \mathrm{k} 1$ and it go through the point py, the straight line is denoted by L4. Get the linear equation, its slope is $-1 / \mathrm{k} 1$ and it go through the point pz, the straight line is denoted by L5;

d) Get the intersection of L4 and L1, L1 and L5, L5 and L2, L2 and L4 respectively, note as A, B, C, D. So the 
rectangle consists of $\mathrm{A}, \mathrm{B}, \mathrm{C}, \mathrm{D}$ is the enclosing rectangle that goes through one side of the convex polygon;

e) Find out all the enclosing rectangles. Select the other edges of the convex polygon, calculate the four vertex coordinates of all the enclosing rectangles and store them repeatedly follow the steps from (a) to (d);

3) Calculate and compare the areas of all the enclosing rectangles of the convex polygon

The enclosing rectangle whose area is minimum is the MERT of the electronic fence. The rectangular ABCD is the minimum enclosing rectangle of the convex polygon. As is shown in Fig. V:

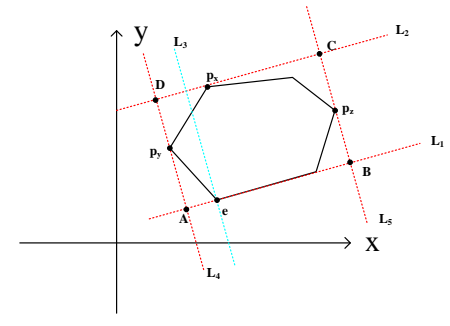

FIGURE V. THE RECT ANGULAR ABCD IS THE MINIMUM ENCLOSING RECT ANGLE OF THE CONVEX POLYGON

It is important to note that the specific strategy to get point py and point pz is plugging all the inflection points of the convex polygon into the linear equation L3 and Classifying them according to whether the left side of the linear equation is more than 0 , there will be two great classes. Then we find out the point to which the distance from the straight line L3 is farthest from the respective classes. That is py and pz. L3: $a x+b y+c=0$

\section{B. The intersection of the tangent line and the MERT}

After obtaining the MERT of the electronic fence, we can make a prediction for whether the UAV will enter the no-fly zone by calculating directly if the tangent line of the current point will intersect with the MERT.

1) Calculate the intersections of the tangent and the lines of four sides of the MERT. Note them as P1, P2, P3, P4. Set the tangent equation: $\mathrm{y}=k * x+b$

Set the linear equations of four sides of the MERT:

$$
\mathrm{y}=k_{i} * x+b_{i}(i=1,2,3,4)
$$

according to the following formula:

$$
\left\{\begin{array}{c}
\mathrm{X}_{\mathrm{i}}=\left(k-k_{i}\right) /\left(b_{\mathrm{i}}-b\right) \\
\mathrm{Y}_{\mathrm{i}}=k^{*} X_{i}+b
\end{array}\right.
$$

By solving the four equation sets consisting of the tangent equation at the current point and the linear equations of four sides of the $M E R T$, respectively, we can get the coordinates (xi, yi) of intersections P1, P2, P3, P4;

2) Judge whether the intersection points are on the sides of the MERT. Since the intersection points are possible on the extension line of the sides of the MERT, so these points which are not on the sides of the rectangle must be eliminated by the constraint conditions. As is shown in the figure above, eliminate P1, P4, hold P2, P3;

3) Select the intersection point to which the distance from the current point of the UAV is nearest from the points that meet the constraint conditions. Take advantage of the formula by which the distance between two points can be gotten to find the nearest point;

4) Calculate the distance between the current point of the $\mathrm{UAV}$ and the nearest points of the sides of the MERT. And that is the distance between the UA V at the current time to the no-fly zone;

\section{SIMULATION EXPERIMENT}

In this paper, the key problem is to solve how to fit with the UA V flight trajectory and how to judge whether the UA V will enter the no-fly zone. These two parts will be verified to illus trate the effectiveness of the algorithm.

\section{A. The FItting of the UAV Flight Trajectory}

Firstly, the discrete coordinate points of the UAV's flight collected for a current time will be transformed into the Gauss plane coordinates, and reflect the movement trend of the UAV by fitting the discrete points into a curve.

Every ten discrete points are selected to execute on a curve fitting in consideration of high real-time performance, which is required by the UAV's Early Warning after many tests. Moreover, in order to improve the reliability of the algorith $\mathrm{m}$, give up the end coordinate point and add the new coordinate point to fit the new curve of flight trajectory again by the least square method while making a new prediction. The two performance are shown as follows:
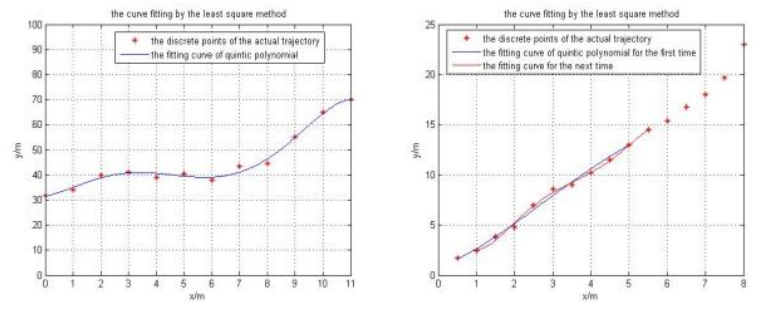

\section{FIGURE VI. THE FITTING CURVE BY THE LEAST SQUARES (A)}

The small red asterisk represents the discrete points in the Gauss-plane rectangular coordinate system are transformed from the coordinates of the GPS. The blue line is the fitting curve of the UAV's flight trajectory by the least squares. It can be seen that the error is small and acceptable. The result states clearly that it is feasible to fit the discrete points of the UAV flight trajectory to predict the movement tendency of the UAV flight.

\section{B. Calculate Whether the UAV Will Enter the No-Fly Zone}

In the simulations, it is imitated how the UAV flies around the no-fly zone in the two-dimensional when overlooked from the sky to the earth in the actual situation. 

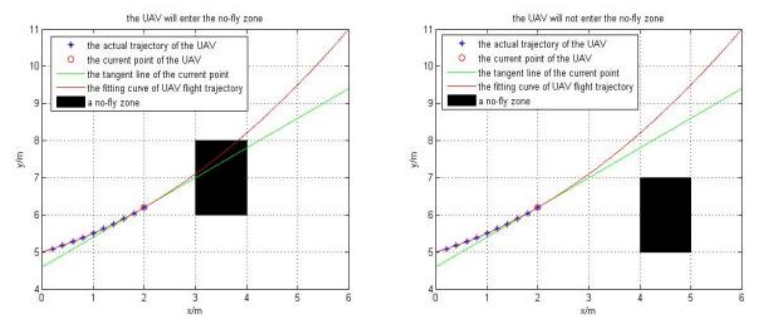

FIGURE VII. THE UAV WILL ENTER(A) AND NOT(B)

The simulation results in FIG VII-A demonstrates that the distance of the UAV to the no-fly zone can be obtained by calculating the intersections that the tangent line intersects with the no-fly zone and selecting the point to which the distance is shortest from the current point of the UAV. If the distance is less than the safe distance, an early warn ing will be sent out. The simulation results in FIG VII-A show that the tangent line of the current point will not intersect with the nofly zone when the fitting curve of the flight trajectory does not intersect with it. It indicates it is relative safe in this situation. There is no need to give an alarm. Therefore it can be done to make the forecast at the next moment. Certainly, in the actual situation, it may occur that the fitting curve intersects with the electronic fence but the tangent line does not intersect with it or the tangent line intersects with the electronic fence but the fitting curve does not intersect with it. If these special cases happen, it is useful to combine the results of prediction before and after in a few moments to consider whether to send an early warning or not. If both at the last moment and at the next mo ment the tangents intersect with the no-fly zone, it indicates at least that at this time the UAV is very likely to enter into the no-fly zone, an early warning should be made in good time to inform the operation personnel of the UAV to change it direction of motion.

\section{CONCLUSION}

In this paper, we proposed NUCLEI. Firstly, we utilize the least squares method to match with the discrete points of the UAV flight trajectory and obtain the fitting curve ( at the next prediction moment, give up the end coordinate point and add the new coordinate to fit the new curve repeatedly ). Secondly, we will calculate whether the tangent line of the fitting curve at the current point will intersect with the electronic fence to predict if the UAV will enter the no-fly zone. If it is, we will calculate the distance from the UAV at the current point to the no-fly zone and then give an alarm timely. If it is not, we will make a prediction of the next moment immediately. At length, aiming at the situation that the shapes of electronic fences that describe the no-fly zone are not fixed, we proposed the MERT to deal with the problem of how to determine whether the UAV will enter a no-fly zone, and then we calculate the shortest distance from the UAV to the no-fly zone. At the same time, the feasibility and effectiveness of the method are verified by the simulation experiments.

\section{REFERENCES}

[1] Wang, B., \& Wang, W. (2015). Application of Uav Remote Sensing in the Now War Milit ary Operations. Wireless Internet Technology.

[2] Zhang, J. (2016). The research of UVA technology application development and regulation. Modern Science \& Technology of Telecommunications, 46(3) 69-71, 78.

[3] Liu, C., Wang, H., Yao, P., et al. (2015). Modeling and analysis of dynamic collision region for UAV avoiding aerial intruders. 41(7) 1231 1238.

[4] Wei, R. X., Lü, M. H., Ru, C. J. and Xu, Z. F. (2014). Reconfiguration collision avoidance method for UAV's formation based on DE-DMPC. XI Tong Gong Cheng Yu Dian Zi Ji Shu/systems Engineering \& Electronics, 36(12) 2473-2478.

[5] Ling, L., Niu, Y. (2015). A particle filter based intruder state estimation method for UAV collision avoidance[C]// Chinese Aut omat ion Congress

[6] Ling, L., Niu, Y., \& Zhu, H. (2015). Lyapunov method-based collision avoidance for UAVs. Chinese Control and Decision Conference.

[7] Ayhan, S., \& Samet, H. (2016). Aircraft Trajectory Prediction Made Easy with Predictive Analytics. ACM SIGKDD International Conference on Knowledge Discovery and Data Mining (pp.21-30). ACM.

[8] W, S. (2015). Trajectory prediction for future air traffic management complex maneuvers and taxiing. Aeronautical Journal -New Series,119(1212) 121-143.

[9] Qing-Fei, X. U., Zhang, X., \& Wei-Min, L. I. (2012). Research and Analy sis of Target Track Prediction Algorithm in 2-Dimensional Region. Avionics Technology.

[10] Zernetsch, S., Kohnen, S., Goldhammer, M., et al. (2016). Trajectory prediction of cyclists using a physical model and an artificial neural net work. IEEE Intelligent Vehicles Symposium(IV), p 833-838.

[11] Xiao-Hao, X. U., Yang, G. Q., \& Liu, J. G. (2001). Comparison of algorithms for air traffic control trajectory prediction.

[12] Shoubei, Y. U., \& Huang, B. J. (2014). 4D flight trajectory prediction model based on improved Kalman filter. Journal of Computer Applications.

[13] Kun, W. U., \& Pan, W. (2007). 4-d trajectory prediction model based on dat a mining. Journal of Computer Applications, 27(11) 2637-2639.

[14] Ma, G. (2004). Application of Data Mining Technology in Predicting the Track of Moving Object. Computer Engineering \& Applications, 40(11) 210-213.

[15] Le, Q. I., \& Zheng, Z. (2016). Trajectory prediction of vessels based on data mining and machine learning. Journal of Digital Information Management.

[16] Feng, Z., \& Zhu, Y. (2016). A survey on trajectory data mining: techniques and applications. IEEE Access, 4, 1-1.

[17] Tian-Qi, G. U., Lei, Z., Shi-Jun, J. I., Tan, X. D., \& Ming, H. U. (2015). Curve fitting method for closed discrete points. Jilin Daxue Xuebao, 45(2) 437-441.

[18] Yao, R., J., Wang, Z., Y., Liang, N. (2016). Kriging comprehensive model optimized by improved least square. Journal Liaoning Technical University(Natural Science), (10) 1159-1 163.

[19] Li, B., L. (2014). Multiple Adaptive Least Square Curve Fitting Algorithm and Applications: [doctoral dissertation]. University of Electronic Science and Technology of China.

[20] Chen, M., J. (2016). Fitting of circular curve based on least square method and it erative method. Science of Surveying and Mapping, 41(1) 194-197,202

[21] Chen, L., Yang, J., Cui, S., Pan, Q., \& Liu, L., I. (2014). Matlab simulation of curve fitting based on least-squares. Journal of Shenyang Normal University, 32(1) 75-79.

[22] Chen, W. J., \& University, J. (2014). The principle of least square method and its application to fitting with experimental curve. Journal of Liaoning Institute of Science \& Technology, 16(4) 33-34,37

[23] Cheng, P. F., Yan, H. W., \& Han, Z. H. (2008). An algorithm for computing the minimum area bounding rectangle of an arbitrary polygon. Journal of Engineering Graphics, 29(1) 122-126.

[24] Pan, W. (2009). New algorithm for convex hull of planer point set. Journal of Chongqing Institute of Technology. 